\title{
Computerized Neurocognitive Battery
}

National Cancer Institute

\section{Source}

National Cancer Institute. Computerized Neurocognitive Battery. NCI Thesaurus. Code C131528.

A series of tests designed to measure performance accuracy and speed on specific neurobehavioral tasks. The included tests measure: abstraction and mental flexibility, attention, working memory, episodic memory (word, face, and spatial recognition memory), language reasoning, spatial processing, sensorimotor, motor speed, and emotion identification. Accuracy is scored as the total number of correct responses and speed is scored as the median response time (RT) for correct items. 